\title{
Zebrafish Tmem230a cooperates with the Delta/Notch signaling pathway to modulate endothelial cell number in angiogenic vessels
}

\begin{tabular}{|c|c|}
\hline Journal: & Journal of Cellular Physiology \\
\hline Manuscript ID & Draft \\
\hline Wiley - Manuscript type: & Original Research Article \\
\hline Date Submitted by the Author: & $\mathrm{n} / \mathrm{a}$ \\
\hline Complete List of Authors: & $\begin{array}{l}\text { Carra, Silvia; University of Milan, Biosciences } \\
\text { Sangiorgio, Lorenzo; University of Milan, Biosciences } \\
\text { Pelucchi, Paride; Istituto di Tecnologie Biomediche Consiglio Nazionale delle } \\
\text { Ricerche, Cancer Stem Cell Research } \\
\text { Cermenati, Solei; University of Milan, Biosciences } \\
\text { Mezzelani, Alessandra; Istituto di Tecnologie Biomediche Consiglio } \\
\text { Nazionale delle Ricerche, Cancer Stem Cell Research } \\
\text { martino, valentina; Istituto di Tecnologie Biomediche Consiglio Nazionale } \\
\text { delle Ricerche, Cancer Stem Cell Research } \\
\text { Palizban, Mira; Istituto di Tecnologie Biomediche Consiglio Nazionale delle } \\
\text { Ricerche, Cancer Stem Cell Research } \\
\text { Albertini, Alberto; Istituto di Tecnologie Biomediche Consiglio Nazionale } \\
\text { delle Ricerche, Cancer Stem Cell Research } \\
\text { goette, martin; University of Münster } \\
\text { Kehler, James; National Institutes of Health } \\
\text { Deflorian, Gianluca; IFOM-IEO Campus } \\
\text { Beltrame, Monica; University of Milan, Biosciences } \\
\text { Giordano, Antonio; Temple University, Sbarro Institute for Cancer Research } \\
\text { and Molecular Medicine, Center for Biotechnology, College of Science and } \\
\text { Technology; University of Siena, Department of Medicine, Surgery and } \\
\text { Neuroscience University of Siena, Siena, Italy } \\
\text { Cotelli, Franco; University of Milano, Biology } \\
\text { reinbold, rolland; Istituto di Tecnologie Biomediche Consiglio Nazionale } \\
\text { delle Ricerche, Cancer Stem Cell Research } \\
\text { Bellipanni, Gianfranco; College of Science and Technology Temple } \\
\text { University, Sbarro Institute for Cancer Research and Molecular Medicine ; } \\
\text { College of Science and Technology Temple University, Department of } \\
\text { Biology } \\
\text { zucchi, ileana; Istituto di Tecnologie Biomediche Consiglio Nazionale delle } \\
\text { Ricerche, Cancer Stem Cell Research }\end{array}$ \\
\hline Key Words: & $\begin{array}{l}\text { Angiogenesis, Delta/Notch signaling, Vegfc/Flt4 signaling, Regenerative } \\
\text { Medicine, blood vessel formation growth }\end{array}$ \\
\hline
\end{tabular}




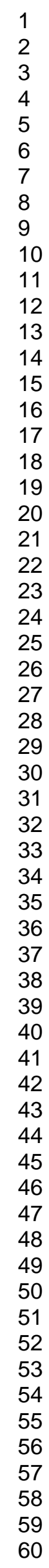

SCHOLARONE $^{\text {th }}$

Manuscripts

John Wiley \& Sons, Inc. 


\section{Zebrafish Tmem230a cooperates with the Delta/Notch signaling pathway to modulate endothelial cell number in angiogenic vessels}

Silvia Carra ${ }^{1,2 \#}$, Lorenzo Sangiorgio ${ }^{1,2 \#}$, Paride Pelucchi ${ }^{2 \#}$, Solei Cermenati ${ }^{1}$, Alessandra Mezzelani $^{2}$, Valentina Martino ${ }^{2}$, Mira Palizban ${ }^{2}$, Alberto Albertini ${ }^{2}$, Martin Götte ${ }^{3}$, James Kehler $^{2,4}$, Gianluca Deflorian ${ }^{5}$, Monica Beltrame ${ }^{1}$, Antonio Giordano ${ }^{6}$, Franco Cotelli ${ }^{1 *}$, Rolland Reinbold ${ }^{2}$, Gianfranco Bellipanni ${ }^{6}$ and Ileana Zucchi ${ }^{2^{*}}$

1. Dipartimento di Bioscienze, Università degli Studi di Milano, Via Celoria 26, Milano 20133, Italy

2. Istituto di Tecnologie Biomediche, Consiglio Nazionale delle Ricerche, via F.1li Cervi 93, Segrate-Milano 20090, Italy

3. Department of Gynecology and Obstetrics, Muenster University Hospital, Muenster D48149, Germany

4. National Institute of Diabetes and Digestive and Kidney Diseases, National Institutes of Health, Bethesda 20814, USA

5. IFOM-IEO Campus, Via Adamello 16, Milano 20139, Italy

6. Center for Biotechnology, Sbarro Institute for Research and Molecular Medicine and Department of Biology, Temple University, Philadelphia 19122, USA

\#First authors with equal contribution

* Corresponding authors with equal contribution

Contact Information:

silvia.carra@unimi.it

lorenzo.sangiorgio@gmail.com paride.pelucchi@itb.cnr.it solei.cermenati@unimi.it alessandra.mezzelani@itb.cnr.it valentina.martino@itb.cnr.it mirapalizban@,hotmail.com alberto.albertini@itb.cnr.it martingotte@uni-muenster.de james.kehler@icloud.com gianluca.deflorian@,ifom.eu monica.beltrame@unimi.it antonio.giordano@temple.edu bellipa4@,temple.edu rolland.reinbold@itb.cnr.it franco.cotelli@,unimi.it ileana.zucchi@itb.cnr.it 


\begin{abstract}
During embryonic development, new arteries and veins form from preexisting vessels in response to specific angiogenic signals. Angiogenic signaling is complex since not all endothelial cells exposed to angiogenic signals respond equally. Some cells will be selected to become tip cells and acquire migration and proliferation capacity necessary for vessel growth while others, the stalk cells become trailer cells that stay connected with pre-existing vessels and act as a linkage to new forming vessels. Additionally, stalk and tip cells have the capacity to interchange their roles. Stalk and tip cellular responses are mediated in part by the interactions of components of the Delta/Notch and Vegf signaling pathways. We have identified in zebrafish, that the transmembrane protein Tmem230a is a novel regulator of angiogenesis by its capacity to regulate the number of the endothelial cells in intersegmental vessels by co-operating with the Delta/Notch signaling pathway. Modulation of Tmem230a expression by itself is sufficient to rescue improper number of endothelial cells induced by aberrant expression or inhibition of the activity of genes associated with the Dll4/Notch pathway in zebrafish. Therefore, Tmem230a may have a modulatory role in vessel-network formation and growth. Our study supports that the activity of Tmem230a is through restricting Vegfe/Flt4 signaling. As the Tmem230 sequence is conserved in human, Tmem230 may represent a promising novel target for drug discovery and for disease therapy and regenerative medicine in promoting or restricting angiogenesis.
\end{abstract}




\section{Introduction}

In embryonic development two distinct processes take place to form the vascular tree. New vessels form de novo for the assembly of mesoderm-derived endothelial precursors (angioblasts) that differentiate into a primitive vascular labyrinth (vasculogenesis). Subsequently, vessel sprouting allows the formation of smaller size vessels (angiogenesis) necessary for growth of a vascular tree necessary creating a network that remodels into arteries and veins(Adams and Alitalo, 2007).

Growth of a vascular tree requires the coordinated control of different functions including proliferation, directional migration and patterning of endothelial cells (ECs). Angiogensis is orchestrated by the regulatory interactions between the vascular endothelial growth factor (Vegf) and Notch signaling pathways that finely control the behavior and positional fate of ECs and determine which cells become tip or stalk behaving cells (Phng and Gerhardt, 2009) (Eilken and Adams, 2010).

The exposure of vessels to pro-angiogenic signals such as Vegf induces the tip cell phenotype only in a fraction of the ECs (Phng and Gerhardt, 2009) (Eilken and Adams, 2010). Tip cell behaviour is strongly controlled by the Notch pathway. Activation of Notch signaling occurs predominantly in stalk cells and takes place by the interaction of Notch with its ligand delta-like 4, D114, leading to the down-regulation of both the Vegfa and Vegfc receptors (Vegfr-2 and Vegfr-3/Flt4, respectively) in these cells (Hellstrom, 2007; Lobov, 2007; Tammela et al., 2008). Therefore, cells with higher levels of D114, low Notch activity and strong Vegf receptor transcription are thought to convert into tip cells. (Phng and Gerhardt, 2009). 
We identified two tmem 230 paralogous genes in zebrafish, tmem230a and tmem230b, and investigated their expression patterns and determined that tmem230a is expressed in the vascular districts in early zebrafish development. Our data reveal that Tmem230a regulates the number of endothelial cells in vessels formed through angiogenic processes by cooperating with the Delta/Notch signalling pathway. In this capacity Tmem230a may have a modulatory role in vessel-network formation and growth.

As only a fraction of the ECs acquires angiogenic behavior required for blood vessel branching, the identification of novel regulators of angiogenesis contributes to a better understanding of both the complex multifaceted regulation of angiogenesis in normal and pathological conditions. Signficantly, as the tmem $230 a$ sequence is conserved in mammals, TMEM230 may represent a new target for human therapy, in promoting or restricting angiogenesis in acute injury and chronic disease, and since blood vessel formation is also required for promoting tumor growth, invasion and metastasis, TMEM230 may also represent a novel target for human cancer therapy.

\section{Materials and Methods}

\section{Zebrafish lines and maintenance.}

Zebrafish (Danio rerio) embryos obtained from natural spawning were raised and maintained according to established techniques(Westerfield, 1993). All experiments with live animals were performed at the University of Milan. All experimental protocols and methods were carried out in accordance with relevant guidelines and regulations of Good Animal Practice approved by the institutional and licensing committee IACUC (Institutional Animal Care and Use Committee) and University of Milan by the Italian 
Decree of March $4^{\text {th }} 2014$, n.26. Embryos were staged according to morphological criteria(Kimmel et al., 1995). Beginning from 24 hpf, embryos were cultured in fish water containing 0.003\% PTU (1-phenyl-2-thiourea; Sigma Aldrich, Saint Louis, Mo, USA) to prevent pigmentation and $0.01 \%$ methylene blue to prevent fungal growth.

The following lines were used: $\mathrm{AB}$ (obtained from the Wilson lab, University College London, London, United Kingdom), $\operatorname{tg}(f l i 1: \mathrm{nEGFP})^{\mathrm{y} 7}$ (Roman et al., 2002), $\operatorname{tg}(f l i 1 \text { :EGFP) })^{\mathrm{y} 1}$ (Lawson, 2002) (from the N.D. Lawson lab, University of Massachusetts Medical School, Boston, USA) and the reporter line Tg(T2KTp1bglob:hmgb1mCherry $)^{\text {jh11 }}$ (from the Argenton Lab, University of Padua, Padua, Italy)(Schiavone et al., 2014) outcrossed with $\operatorname{tg}(f l i 1: \text { EGFP })^{\mathrm{y} 1}$.

\section{Tmem 230 sequence analysis.}

Sequence analysis was performed using Genomic Database (www.ensembl.org) NCBI (http://www.ncbi.nlm.nih.gov/BLAST/) ClustalW (http://www.ebi.ac.uk/Tools/clustalw/) Genomicus (http://www.genomicus.biologie.ens.fr/genomicus-83.01/cgi-bin/search.pl). Prediction of transmembrane regions, topology and orientation analysis was performed using TMPRED (http://www.ch.embnet.org/software/TMPRED_form.html)(Hofmann, 1993) and HMMTOP (http://www.enzim.hu/hmmtop/index.php)(Tusnady and Simon, 2001).

\section{Expression pattern analysis.}

RT-PCR (Reverse Transcription-Polymerase Chain Reaction) was performed on total RNA prepared from zebrafish oocytes and embryos at different developmental stages using the Totally RNA Isolation Kit (Ambion, ThermoFisher, Waltham MA, USA) or the 
RNAgents Total RNA Isolation System (Promega, Madison, WI, USA), treated with DNase I RNase free (Roche, Basel, Switzerland) to avoid possible contamination from genomic DNA and then reverse transcribed using the ImProm-II Reverse Transcription System (Promega) and random primers. The cDNAs were then PCR amplified using GOTaq polymerase (Promega).

The following PCR primers were used: tmem230a for: 5'GCAGAGGATCGAGCAGTGTT 3', rev: 5'GAAGGCAACACATGCAACAG 3', tmem230b left: AGAAGATGCCTGCTCGAAGC 3', tmem230b right: 5' GCTGAGATCTCTGTCAGTCG 3'. Specific $\beta \square$ actin primers were used as internal control to check cDNA quality and possible genomic contamination(Argenton et al., 2004).

\section{In situ hybridization and imaging.}

Whole-mount in situ hybridization (WISH) was performed as described(Thisse et al., 1993; Wu et al., 2011). For tmem230a and tmem $230 b$ probe preparations, templates spanning the last portion of coding sequence and the 3' UTR region for tmem230a or the entire coding sequence for tmem $230 b$ were generated by RT-PCR on total RNA extracted from $26 \mathrm{hpf}$ embryos using the following primers: tmem230a newlF: 5, GCTTCCAAAGGTTACCGTGG 3' tmem230a new2R: 5' AAAGGCTTGGACACATCTGC 3' $\quad$ tmem230b left: AGAAGATGCCTGCTCGAAGC 3' tmem230b right:

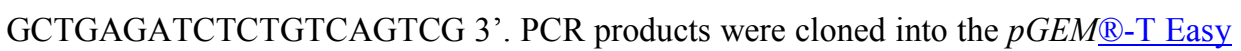
vector (Promega). 
The cDNA-containing plasmids were linearized and transcribed with T7 and Sp6 RNA polymerase (Roche) for antisense and sense riboprobe synthesis.

Plasmid probes for flk1(Fouquet et al., 1997), flt4(Thompson et al., 1998), and dll4(Siekmann and Lawson, 2007) were kindly provided by N.D Lawson, and efnb2a and ephB4(Lawson et al., 2001) were kindly provided by R. Patient (Weatherall Institute of Molecular Medicine, University of Oxford, John Radcliffe Hospital, Oxford, U.K.). Images of stained embryos were taken with a Leica MZFLIII epifluorescence stereomicroscope equipped with a DFC 480-R2 digital camera using the LAS imaging software (Leica, Germany).

For histological sections, stained embryos were re-fixed in 4\% PFA, dehydrated, wax embedded, sectioned ( $8 \mu \mathrm{m})$ by a microtome (Leitz 1516) and stained with eosin. Images were taken with a Leica DM6000 B microscope equipped with a Leica 480 digital camera using the LAS software (Leica, Germany).

\section{Morpholino and mRNA injections and detection of splice variants of the tmem $230 a$ transcript by RT-PCR.}

Two different antisense morpholinos (MOs) for tmem230a were synthesized by Gene Tools (Philomath, OR, USA): tmem230a-MO1 5, GTGTTGTTCGGGTTGCCATCATA $3^{\prime}$ and tmem230a-MO2 5' CAGCTTAGATATTTTCTCACCTGTA 3'. tmem230a-MO1 was designed on the region surrounding the AUG translation start codon of the transcript. tmem230a-MO2 was designed on the exon2/intron 2 boundary. The following morpholino was already described: dll4-MO(Hogan, 2009). As a control for unspecific effects, each experiment 
was performed in parallel with a standard control morpholino control (std-MO) that has no target in zebrafish. All morpholinos were diluted in Danieau's solution(Nasevicius and Ekker, 2000) and injected at the 1-2 cell stage embryo. Rhodamine dextran (Molecular Probes) was usually co-injected as a tracer. After injection, embryos were raised in fish water (previously described) at $28^{\circ} \mathrm{C}$ and observed up to the stage of interest. For a better observation, the injected embryos were anaesthetized using $0.016 \%$ tricaine (Ethyl 3aminobenzoate methanesulfonate salt, Sigma Aldrich) in fish water. To assess the ideal concentration of morpholino we injected several dilutions and verified at $24 \mathrm{hpf}$ the overall effects of the morpholino on embryo phenotype. The injection of tmem230a-MO1 at a concentration above or equal to $0.4 \mathrm{pmol} /$ embryo led to morphological defects, such as head defects and bent tail (not shown), and increased mortality in a dose dependent manner (Supplementary Fig. S4a), suggesting the activation of unspecific mechanisms at those concentrations. While, at a concentration of $0.3 \mathrm{pmol} / \mathrm{embryo}$ and below the survival rate was high and the embryos had a normal morphology and development (Supplementary Fig. S4a). We proceded in a similar way to determine the concentration of splicing morpholino tmem 230a-MO2 to inject and we found we could inject an higher dose (1pmol/embryo) of this morpholino with not apparent effects in the gross morphology of the embryo (Supplementary Fig S4b).

Images were acquired using a Leica MZFLIII epifluorescence stereomicroscope equipped with a DFC 480-R2 digital camera and the LAS imaging software (Leica, Germany).

At 29 hpf, total RNA was extracted from embryos injected with tmem $230 a-\mathrm{MO} 2$ or stdMO with RNAgents Total RNA Isolation System (Promega). Reverse transcription was carried out with the ImProm-II Reverse Transcription System (Promega) and random 
primers. PCR was performed to detect splice variants of tmem 230a. The following primers were used: tmem 230a for: 5'GCAGAGGATCGAGCAGTGTT 3' and tmem230a rev: 5'GAAGGCAACACATGCAACAG 3'. RT-PCR products were then sequenced.

For tmem $230 a$ mRNA, the complete coding sequence was cloned into the $\mathrm{pCS}^{+}$plasmid which was then digested with NotI and in vitro transcribed using the mMESSAGE mMACHINE kit (Ambion). Rescue experiments were performed with the co-injection of 1pmol/embryo tmem230a-MO2 and $400 \mathrm{pg} / \mathrm{embryo}$ tmem $230 a$ mRNA diluted in the Danieau's solution into 1-cell stage embryos.

\section{Analysis of ISV cell number.}

We scored ISV cell number by counting cell nuclei expressing the GFP in $\operatorname{tg}(\text { fli1:nEGFP })^{\mathrm{y} 7}$ embryos at 29 hpf. We considered for our analysis the first 10 intersegmental vessels anterior to the anus. We mounted injected embryos of each sample in $1 \%$ low-melting agarose (adding some drops of tricaine as anaesthetic) and observed in every embryo the same group of ISVs. Images were taken with a confocal Leica TCS SP2 AOBS microscope, equipped with an argon laser, with a PL FLUOTAR 20X x 0.50 NA objective. The mean of the cell number was calculated by counting GFP positive nuclei in 10 segments/embryo for the number of embryos indicated by $\mathrm{n}$. Standard error of mean (SEM) is indicated as vertical bars with caps.

\section{DAPT treatment.}

A $40 \mathrm{mM}$ stock solution of DAPT ( $N$-[N-(3,5-difluorophenacetyl-1-alanyl)]-Sphenylglycine $t$-butyl ester, $\gamma$-secretase inhibitor IX; Calbiochem, La Jolla, CA, USA) 
was diluted to a concentration of $200 \mu \mathrm{M}$ in E3 embryo medium as described(Westerfield, 1993). tmem230a mRNA and std-MO injected embryos were dechorionated by pronase (Sigma Aldrich) treatment(Westerfield, 1993) and treated with DAPT from $17 \mathrm{hpf}$ to $29 \mathrm{hpf}$ at $28^{\circ} \mathrm{C}$. As DAPT is prepared with DMSO, tmem $230 a$ mRNA and std-MO injected embryos were treated with E3 embryo medium containing the same concentration of DMSO used for the DAPT treated embryos.

\section{Live-imaging analysis of Notch reporter expression.}

To assay the capacity of Tmem230a to regulate Notch signalling, we injected tmem230aMO1 into 1-cell stage embryos generated from crossing the transgenic line $\operatorname{Tg}$ (T2KTplbglob:hmgb1-mCherry) with $\operatorname{Tg}\left(\right.$ flila:EGFP $^{y 1}$. The level of mCherry fluorescence expression in the vessels of the embryos was scored by confocal microscopic analysis. We mounted five embryos at $48 \mathrm{hpf}$ from each sample in $1.2 \%$ low-melting agarose (adding some drops of tricaine as anaesthetic) and observed in every embryo the same group of 3 ISVs up to the yolk extension. Images were taken with a Leica TCS SP2 confocal microscope, using a water-immersion objective 40X.

\section{Statistical analysis.}

Statistical analysis was performed with one-way ANOVA analysis of variance technique and with Dunnett's post test using GraphPad PRISM versions 5.0 and 6.0 (GraphPad, San Diego, CA, USA). In the graphs, ${ }^{*}$ and $* *$ mark statistically significant data with a $\mathrm{p}$ value $<0.05$ and $<0.01$, respectively. Statistically highly significant data, with a $p$ value $<0.001$, are marked by $* * *$. 


\section{Results}

\section{Tmem 230 identification and bioinformatic analysis}

We have identified Tmem 230 differentially expressed at the onset of in vitro differentiation of pluripotent mouse embryonic stem cells (mESCs) (manuscript in preparation, PP and RR). To identify the role of Tmem230 in the earliest stages of vertebrate embryogenesis, Tmem230 expression was modulated in zebrafish, as a model for embryo tissue and organ development.

The zebrafish genome encodes two tmem230 genes, tmem230a (zgc:101123) on chromosome 10 and its paralogue tmem230b (zgc:162251) on chromosome 8 . The analysis of tmem 230 genes across vertebrates revealed that both genes are orthologs of mammalian Tmem230 and both genes may have arisen from a duplication event (Fig. 1a). The tmem230a and $b$ transcripts are 1663 and 1019 bp in length and encode proteins of 120 and 115 amino acids, respectively (Fig. 1b). The Tmem proteins are highly related to each other ( $83 \%$ identity, Supplementary Fig. S1a). In addition, Tmem230a protein shares $76 \%$ identity with their respective human and mouse orthologs, while Tmem $230 \mathrm{~b}$ shares $74 \%$ identity with the human and $75 \%$ with the mouse ortholog, respectively. Transmembrane topology prediction analysis of conserved domains revealed that Tmem230a and b proteins contain 2 transmembrane domains (Supplementary Fig. S1b). 


\section{tmem 230a is expressed in blood vessels during embryonic and early larval zebrafish} development.

Temporal expression of both zebrafish tmem 230 genes $(a$ and $b$ ) was analyzed in embryonic and early larval development and in adult organs and tissues by RT-PCR analysis (Fig. 2a and Supplementary Fig. S2a). Both transcripts were detected at all analyzed stages from cleavage up to $5 \mathrm{dpf}$, as well as in oocytes, suggesting that both transcripts are both maternally and zygotically expressed. Furthermore, tmem230a and tmem $230 \mathrm{~b}$ expression was detected in all adult organs and tissues tested (brain, eyes, gills, gut, heart, liver and muscle). Whole-mount in situ hybridization (WISH) analysis revealed that from the mid somitogenesis stage (15 somites) to $2 \mathrm{dpf}$, tmem $230 a$ has higher expression in the developing vascular system than tmem $230 b$ (Fig. 2b-j and Supplementary Fig. S2b-f). At the 15 somite stage, tmem $230 a$ was expressed in the telencephalon, mesencephalon and hindbrain, and was starting to be expressed in the Intermediate Cell Mass (ICM) and in the forming axial vasculature (Fig. 2b). At 26 hpf, tmem230a was expressed in the pharyngeal arch mesenchyme, in the Dorsal Aorta (DA) and Caudal Vein (CV) (Fig. 2c,c',d,h,i). At 2 dpf, a strong hybridization signal was detected at the level of the mandibular arches and in the fin buds, and moreover tmem230a was expressed at low levels in the CV plexus region (Fig. 2e,f,g,j). In contrast, no hybridization signal was detected for tmem $230 \mathrm{~b}$ in the forming vasculature (Supplementary Fig. S2b-f). During somitogenesis (15 to 20 somite stages), WISH staining revealed a faint and ubiquitous tmem $230 b$ hybridization signal (Supplementary Fig. S2b,c). At the 15 somite stage, tmem $230 b$ expression appeared fairly widespread, while at the 20 somite stage was expressed in the tail and eyes. At 26 hpf, tmem $230 \mathrm{~b}$ was 
detectable in the optic tectum, mesencephalon, hindbrain and in the tail including the $\mathrm{CV}$ region and in the somites (Supplementary Fig. S2d, d',e). At 2 dpf, tmem $230 b$ was only slightly expressed in the mesencephalon (Supplementary Fig. S2f).

Since our data indicate a robust tmem230a expression in the developing vasculature not overlapping with the expression of tmem230b, we investigated the role of Tmem230a in vascular development.

\section{Tmem230a modulates ISV endothelial cell number.}

To determine the role of tmem $230 a$, we first looked to the effects of tmem230a knockdown, to reduce Tmem230a protein in the developing vasculature. To not interfere with the early embryonic expression of tmem $230 a$, a gene knockdown approach with morpholinos was used. Knockdown experiments were performed by independent injections of two different morpholinos: a low dose tmem230a-MO1 and tmem230aMO2, designed to block mRNA translation or splicing, respectively (Fig. 3). The sequences of both tmem230a-morpholinos were analyzed to exclude cross targeting to tmem230b (Supplementary Fig. S3a). We injected tmem230a-MO1 at the selected dose of $0.3 \mathrm{pmol} / \mathrm{embryo}$ and tmem $230 a-\mathrm{MO} 2$ at $1 \mathrm{pmol} / \mathrm{embryo}$ in one cell stage $\operatorname{tg}(f l i 1: \mathrm{nEGFP})^{\mathrm{y} 7}$ embryos. These concentrations of morpholinos did not affect the gross phenotype of the zebrafish embryos and the main axial vessels appeared normal in all injected embryos at $29 \mathrm{hpf}$ and $2 \mathrm{dpf}$ (Fig. 3 and see Methods and Supplementary Figs. $\mathrm{S} 4$ and S5). The injections in transgenic embryos $\operatorname{tg}(f l i 1: \mathrm{nEGFP})^{\mathrm{y} 7}$ allowed for the quantification of intersegmental vessel (ISV) cell numbers by counting cell nuclei expressing the green fluorescent protein (GFP) (Fig. $3 \mathrm{a}$-g). We counted cells in the first 10 intersegmental vessels anterior to the anus and found a statistically significant increase 
of the mean number of cells in ISVs when $\operatorname{tg}(f l i 1: \mathrm{nEGFP})^{\mathrm{y} 7}$ embryos were injected with 0.3 pmol MO1 $(\mathrm{n}=100)$ compared to control std-MO injected embryos $(\mathrm{n}=69)$ (Fig. $3 \mathrm{e}, \mathrm{k})($ Roman et al., 2002). To further confirm these results, when we injected the spliceblocking tmem230a-MO2 at $1 \mathrm{pmol} / \mathrm{embryo}$ in the same transgenic line embryos, we observed a comparable increase in ISV cell number though with a different penetrance (Supplementary Fig. S4b and Fig. 3e,i,1). The injection of tmem230a-MO2 which targets the exon2-intron2 boundary, generated a transcript unable to splice exon 2 from intron 2 (Supplementary Fig. S3b). Sequencing of the smaller PCR product confirmed a sequence skipping tmem $230 a$ exon 2 consistent with a previous report showing that targeting of a E2/I2 junction may result in exon 2 skipping (Morcos, 2007). This mRNA having no starting codon in frame with the tmem230a sequence yields a non-functional tmem $230 a$ mRNA.

In parallel to the loss-of-function experiments, mRNA over-expression experiments were performed. We injected tmem $230 a$ mRNA (400 pg/embryo) in one cell stage $\operatorname{tg}(f l i 1: \mathrm{nEGFP})^{\mathrm{y} 7}$ embryos. As for the knock-down experiments, we counted the number of ISV cells present in the first 10 intersegmental vessels anterior to the anus. Consistent with the phenotype observed with the loss-of-function of Tmem230a, the gain-offunction experiment resulted into the opposite phenotype. We found a statistically significant $(\mathrm{p}<0.0001)$ decrease of the mean number of cells in ISVs in the tmem230aover-expressing embryos (29.17 mean ISV cells present in the first 10 intersegmental vessels anterior to the anus for the tmem230a over-expressing embryos, $n=60$, versus 35.06 mean cell number for the control, $n=32$ ), (Fig. $3 \mathrm{j}, \mathrm{k}$ ). 
Experiments using two independent morpholinos at concentrations that did not result in gross morphological defects, both gave the same in vivo phenotype affecting the ISV cell number. In addition, Tmem230a gain and loss of function experiments consistently produced opposite effects on the ISV cell number. Moreover, we fully rescued the effects on ISV cell number of injections of $1 \mathrm{pmol} / \mathrm{embryo}$ of tmem230a-MO2 by tmem230a mRNA over-expression (400 pg/embryo) (Fig. 31).

Taken together these data suggest a bona fide role of tmem230a in regulating ISV cell number during angiogenesis. Considering that both morpholinos produced the same phenotypes, we performed all following experiments by injecting tmem $230 \mathrm{a}$-MO1 into embryos, which we indicate as tmem230a morphants.

\section{Tmem230a knockdown affects angiogenic blood vessel growth rather than artero- venous endothelial cell fate.}

The effect of Tmem230a in ISV cell number could be explained in two possible ways: either Tmem230a affects blood vessel growth or regulates artero-venous cell fate switch. To gain insight into the molecular events following tmem230a-MO1 injection, we analyzed the expression of different vascular markers: ephrin-B2 (efnb2a) and its receptor ephB4, specifically expressed in arterial and venous endothelium, and flkl (vegf receptor 2, vegfr2), flt4 (vegf receptor 3, vegfr3) and dll4 (notch ligand delta-like 4) which are preferentially expressed in tip rather than in stalk cells(Adams and Alitalo, 2007; Blanco and Gerhardt, 2013; Fouquet et al., 1997; Gerety et al., 1999; Lawson et al., 2001; Phng and Gerhardt, 2009; Shutter et al., 2000; Siekmann and Lawson, 2007; 
Thompson et al., 1998; Wang, 1998). In std-MO and tmem230a-MO1 injected embryos, efnb2a and ephb4 expression levels were comparable (for efnb2a $\mathrm{n}=74, \mathrm{n}=75$ respectively and for ephb4 $\mathrm{n}=77, \mathrm{n}=76$, respectively; Fig. $4 \mathrm{a}-\mathrm{d}$ ). In contrast, increase of $f l k 1, f l t 4$, and dll4 staining in ISVs and in the Dorsal Longitudinal Anastomotic Vessels (DLAVs) was observed in tmem $230 a$ morphants (for $f l k 183 \% \mathrm{n}=57$; for $f l t 479 \% \mathrm{n}=52$; and for $d l l 453 \% \mathrm{n}=44$, respectively) compared with control embryos (for $f l k 1 \mathrm{n}=16$; for flt $4 \mathrm{n}=19$; and for dll4 $\mathrm{n}=18$; Fig. 4e-j).

No change in efnb2a and ephB4, but increase in $f l k 1, f l t 4$, and dll4 marker levels in tmem230a morphants strongly suggests that more ISV cells were generated by tmem230a-MO1 injection. These results provided support that the role of tmem230a is restricted to angiogenic blood vessel growth and that tmem230a promotes angiogenic cell behavior rather than the determination of artero-venous cell fates. The increase of cell number can be due to various cellular processes including increase in cell proliferation and/or a result of cellular migration of ISV cells from the aorta.

\section{Tmem230a and the Notch/Delta signaling pathway cooperate in modulating ISV endothelial cell number.}

Previous studies have demonstrated that Notch restricts angiogenesis and that loss of the Notch ligand dll4 led to supernumerary endothelial cells within the ISA(Leslie et al., 2007; Siekmann and Lawson, 2007). As tmem230a mRNA over-expression reduced ISV cell number, we wanted to determine whether tmem230a is involved in the Notch signaling pathway, and whether it acts synergistically with dll4 in regulating the ISV cell number. Two independent approaches were used to answer these questions. First, we co- 
injected into embryos subcritical doses of tmem230a- (0.07 pmol/embryo) and dll4- (0.09 pmol/embryo) MOs (Fig. 5a-f). Results show that the subcritical doses of each morpholino do not cause alterations in ISV cell number when injected separately (Fig. 5e). However, when subcritical doses of both dll4- and tmem230a-MOs were co-injected into the same embryos a statistically significant increase in ISV cell number was observed, suggesting a synergistic effect of D114 and Tmem230a (Fig. 5c,e). Consistent with a role of Tmem230a in regulating ISV cell number, co-injection of tmem230a mRNA (400 pg/embryo) together with dll4-MO (0.4 pmol/embryo) rescued normal endothelial cell numbers in ISVs (Fig. 5d,f). As Dll4 is part of the Notch pathway and Notch restricts angiogenesis we hypothesized that a second and independent way to block Notch signaling would also produce the same results. Therefore, we investigated the effect of tmem230a in a context where the Notch signaling was blocked by using the $\gamma$ secretase inhibitor, DAPT(Geling et al., 2002). Consistent with the results obtained with the dll4-morphants, embryos treated with $200 \mu \mathrm{M}$ DAPT showed an increase in the number of ISV cells compared to DMSO-control treated embryos (Fig. 5g). Embryos injected with tmem230a mRNA and treated with DAPT showed a number of ISV cells comparable to that of std-MO injected control embryos treated with DMSO, while DMSO-control embryos injected with tmem $230 a$ mRNA showed a decrease in ISV cell number as previously seen (Fig. $3 \mathrm{j}, \mathrm{k}$ ). These results confirm that embryos overexpressing tmem230a can rescue back the correct number of ISV cells in Notch signaling blocked embryos (Fig. 5g). Taken together, these data are consistent with the hypothesis that Tmem230a acts synergistically with D114 and has a role mediating Notch signaling pathway for ISV development. 
We further tested the effect of Tmem230a down regulation in Notch-responsive vessels in vivo. We injected tmem230a-MO1 into embryos obtained by the outcross of the transgenic line $\operatorname{tg}(f l i 1: \mathrm{EGFP})^{\mathrm{y} 1}$ with the transgenic line $\operatorname{tg}($ T2KTp1bglob:hmgb1$m$ Cherry $)^{\text {jh11 }}$ which expresses mCherry in tissues known to be Notch responsive. Expression of nuclear mCherry fluorescence protein occurs when the Notch intra-cellular domain (NICD) and its cofactor RBP-JK bind to the promoter of the Epstein Barr Virus terminal protein 1 (TP1) gene which contains two Rbp-J火 binding sites(Parsons et al., 2009; Schiavone et al., 2014). For a better visualization of the activated hmgb1-mCherry we analyzed embryos at $48 \mathrm{hpf}$ instead of the $29 \mathrm{hpf}$ time point used for our previous experiments.

At 48 hpf, 76\% $(\mathrm{n}=98)$ of tmem $230 a$ morphants showed decrease of mCherry expression in ISVs and DA with respect to std-MO injected embryos $(n=125)$ (Fig. 6), strongly suggesting the involvement of Tmem230a in the positive modulation of Notch signaling in vascular districts responsive to Notch.

All results presented here support that Tmem230a has a modulatory role in the D114/Notch signaling pathway to limit angiogenic cell behavior.

\section{Discussion}

In zebrafish, embryonic trunk angiogenesis takes place in two distinct waves: formation of primary and secondary sprouts. From about 20 hpf, primary sprouts bilaterally form from the DA to give rise to intersegmental arterial (ISA) vessels, which are completed by 1.5 dpf. From about $32 \mathrm{hpf}$, secondary sprouts emerge from the posterior cardinal vein (PCV) to give rise to venous ISVs and lymphatic vascular precursors(Childs et al., 2002; 
Isogai et al., 2003; Yaniv et al., 2006). At 29-30 hpf, ISVs consist of three or four endothelial cells with different positional fates: a tip cell that is the dorsal-most T-shaped cell that contributes to the DLAV, an adjacent connector cell which is situated along the length of the medial somite boundary, and a base cell connected to the DA(Siekmann and Lawson, 2007). Growth of intersegmental veins requires the coordination of tip and stalk cell behaviors with different capacities for proliferation, directional migration, patterning and positional fates of ECs.

Here, we identified two tmem 230 paralogous genes in zebrafish. In this work, we investigated the expression pattern and the function of Tmem230a.

As our preliminary data (Fig. 2) indicated that tmem230a may have diverse functions since it was expressed throughout embryonic development including pre-gastrulation development, to focus only on the role of tmem230a in vascular development we decided to modulate tmem230a levels by morpholinos. In contrast to morpholinos, transgenic technologies and use of the CRISPR-Cas9 approach to induce mutations could produce early phenotypes, thus, mask later developmental events, like the vascular phenotype that we were interested to study (Blum et al., 2015). Injections of two independent tmem230aMOs for the down regulation of Tmem230a led to the increase of endothelial cell number within the ISVs. Interestingly, over-expression of tmem $230 \mathrm{a}$ mRNA resulted into the opposite phenotype, a marked decrease of endothelial cell number within the ISVs, supporting that the role of Tmem230a is to limit endothelial cell number and therefore modulate angiogenic vessel growth. 
The fact that two independent morpholinos resulted into the same phenotype and that the tmem230a-MO phenotype was rescued by tmem230a mRNA injection, demonstrated the specificity of the tmem $230 a$ morpholino obtained phenotypes.

The increase in number of ISV cells could be due to several events, such as increase in cell proliferation or a result of cellular migration of ISV endothelial cells from aorta which could be generally grouped in two main possibilities, changes in antero-venus cell fate or changes in precursors cell number. We showed here that flkl, flt4, and dll4 staining in ISVs and in the Dorsal Longitudinal Anastomotic Vessels (DLAVs) increased and no difference in the arterial and venous marker expression were observed in tmem230a morphants compared to control embryos, suggesting that tmem230a does not regulate artero-venous cell fate specification but modulates the EC number in angiogenic blood vessels. It would be interesting to determine if these cells are generated by increased proliferation of local ISV cells or migration of ISV cells from aorta. We are planning to address this question in future work.

Different signals regulate tip and stalk cell behaviors by the interactions of genes associated to the Vegf and Notch signaling pathways(Eilken and Adams, 2010; Phng and Gerhardt, 2009). As the tmem230a morphants displayed the same ISV phenotype observed with dll4 knockdown experiments previously described(Siekmann and Lawson, 2007), we investigated whether Tmem230a cooperates with, and/or modulates the Dl14/Notch signaling pathway in regulating angiogenesis. The co-injection of subcritical doses of tmem230a-MO1 and dll4-MO demonstrated that the down regulation of the two genes resulted in a synergistic increase of the ISV cell number, while the subcritical dose of tmem230a-MO1 or dll4-MO when injected individually, did not promote change in the 
number of ISV cells. Consistent with the subcritical co-injection results, a dose of dll4MO higher than subcritical resulted in a supernumerary ISV cell number, whereas the tmem230a mRNA and dll4-MO co-injection rescued the phenotype generated by the dll4MO higher dose. To further confirm the morpholino experiments, we decided to chemically block Notch signaling with DAPT treatment. The treated embryos displayed an excessive ISV cell number. In absence of Dl14 (dll4-MO) or with inhibition of Notch (NICD release with DAPT treatment), the injection of tmem230 mRNA rescued the excessive ISV cell number, suggesting that tmem $230 a$ mRNA can independently compensate for the knockdown of dll4 and inhibition of Notch. In agreement, decrease of mCherry expression under the control of Notch responsive elements was observed in vessels in tmem $230 a$ morphants supporting the involvement of tmem230a, like that for dll4 in the positive modulation of Notch signaling.

These results strongly support that tmem230a has a role Dl14/Notch signaling. However, the precise epistatic relationships of tmem230a with respect the D114/Notch signaling in the modulation of EC numbers in ISVs still needs to be determined. In fact, our experiments suggest that tmem $230 a$ can compensate and/or cooperate with both the Dl14/Notch signaling pathways in limiting the number of endothelial cells in angiogenic processes in early development of zebrafish. Moreover, it still remains to be determined which components of the D114/Notch signaling pathways Tmem230a interacts with. For instance, does Tmem230a work with Notch as a co-receptor for Delta or cooperate with Notch within the cytoplasm for signal transduction. In order to answer these questions, co-localization of Tmem230a with D114/Notch signaling components need to be carried out. 
Our study is the first to identify TMEM230 as a novel regulator and modulator of angiogenesis associated blood vessel-network formation and growth. Recently, TMEM230 has been identified when mutated to have a role in Parkinson's disease. As to how TMEM230 mutation(s) contributes at the molecular level to the Parkinson's phenotype is still under investigation.(Baumann et al., 2017; Deng et al., 2016; Giri et al., 2017; He et al., 2016; Kim et al., 2017; Olszewska et al., 2016; Quadri et al., 2017; Wu et al., 2016; Yan et al., 2017). Our study is the first to associate and characterize TMEM230 to specific signaling pathways and provides first insight into how TMEM230 functions at the molecular level. Additionally, our study and the recent discovery of role of TMEM230 in Parkinson's disease suggest that novel genes with tantalizingly multiple functions in normal and disease vertebrate development are still to be discovered.

As the tmem230a gene sequence is conserved in vertebrates including human, and modulation of tmem $230 a$ expression alone was sufficient to rescue improper number of endothelial cells induced by aberrant expression levels of genes or by inhibition of gene activity in the Dl14/Notch pathway, this suggests that the TMEM230 protein is a novel and potentially clinically important alternative target for human regenerative therapy. For instance, extended modulation of TMEM230 expression by pharmacological agents may allow for inducing or mitigating new blood vessel formation for promoting following acute injury or restricting such as for macular degeneration in angiogenesis. As blood vessel formation is also essential for promoting tumor growth, circulation of cancer cells 
and metastasis, TMEM230 protein may also be a target for cancer therapy by repressing new blood vessel growth.

\section{Declaration of conflict of Interest.}

The authors declare that a patent application was submitted utilizing TMEM230 as a novel gene for regulation of human angiogenesis. Reinbold RA., Zucchi I. Nuovi regolatori dell'angiogenesi, patent application pending.

\section{Acknowledgements}

We like to thank Professor Federico Bussolino (University of Turin, Italy) for critical suggestions and Natascia Tiso (Argenton Lab, University of Padua, Padua, Italy) for providing the $\operatorname{tg}(\mathrm{T} 2 \mathrm{KTp} 1 \mathrm{bglob}: \mathrm{hmgb} 1-\mathrm{mCherry})^{\mathrm{jh} 11}$ transgenic line. Funding for this research is provided to IZ: Cariplo Progetti-Internazionali $2008-2015$ by Fondazione Cariplo; grants RBAP11BYNP and RBAP11Z4Z9 by MIUR-FIRB; and Progetto Bandiera Interomics. Silvia Carra, Lorenzo Sangiorgio and James Kehler were supported by fellowships from the Fondazione Cariplo to IZ.

\section{Authors' Contributions}

SiCa, LS, and SoCe performed the zebrafish experiments and data analysis; PP, AM, VM and MP contributed tools for the experiments; GD performed experiments with the transgenic line $\operatorname{tg}(\mathrm{T} 2 \mathrm{KT} \text { plbglob:hmgb1-mCherry })^{\mathrm{jh} 11}$ crossed with the transgenic line 


\section{Literature Cited}

Adams RH, Alitalo K. 2007. Molecular regulation of angiogenesis and lymphangiogenesis. Nat Rev Mol Cell Biol 8(6):464-478.

Argenton F, Giudici S, Deflorian G, Cimbro S, Cotelli F, Beltrame M. 2004. Ectopic expression and knockdown of a zebrafish sox 21 reveal its role as a transcriptional repressor in early development. Mech Dev 121(2):131-142.

Baumann H, Wolff S, Munchau A, Hagenah JM, Lohmann K, Klein C. 2017. Evaluating the role of TMEM230 variants in Parkinson's disease. Parkinsonism Relat Disord 35:100-101.

Blanco R, Gerhardt H. 2013. VEGF and Notch in tip and stalk cell selection. Cold Spring Harb Perspect Med 3(1):a006569.

Blum M, De Robertis EM, Wallingford JB, Niehrs C. 2015. Morpholinos: Antisense and Sensibility. Dev Cell 35(2):145-149.

Childs S, Chen JN, Garrity DM, Fishman MC. 2002. Patterning of angiogenesis in the zebrafish embryo. Development 129(4):973-982.

Deng HX, Shi Y, Yang Y, Ahmeti KB, Miller N, Huang C, Cheng L, Zhai H, Deng S, Nuytemans K, Corbett NJ, Kim MJ, Deng H, Tang B, Yang Z, Xu Y, Chan P, Huang B, Gao XP, Song Z, Liu Z, Fecto F, Siddique N, Foroud T, Jankovic J, Ghetti B, Nicholson DA, Krainc D, Melen O, Vance JM, Pericak-Vance MA, Ma YC, Rajput AH, Siddique T. 2016. Identification of TMEM230 mutations in familial Parkinson's disease. Nat Genet 48(7):733-739.

Eilken HM, Adams RH. 2010. Dynamics of endothelial cell behavior in sprouting angiogenesis. Curr Opin Cell Biol 22(5):617-625. 
Fouquet B, Weinstein BM, Serluca FC, Fishman MC. 1997. Vessel patterning in the embryo of the zebrafish: guidance by notochord. Dev Biol 183(1):37-48.

Geling A, Steiner H, Willem M, Bally-Cuif L, Haass C. 2002. A gamma-secretase inhibitor blocks Notch signaling in vivo and causes a severe neurogenic phenotype in zebrafish. EMBO Rep 3(7):688-694.

Gerety SS, Wang HU, Chen ZF, Anderson DJ. 1999. Symmetrical mutant phenotypes of the receptor EphB4 and its specific transmembrane ligand ephrin-B2 in cardiovascular development. Mol Cell 4(3):403-414.

Giri A, Mok KY, Jansen I, Sharma M, Tesson C, Mangone G, Lesage S, Bras JM, Shulman JM, Sheerin UM, International Parkinson's Disease C, Diez-Fairen M, Pastor P, Marti MJ, Ezquerra M, Tolosa E, Correia-Guedes L, Ferreira J, Amin N, van Duijn CM, van Rooij J, Uitterlinden AG, Kraaij R, Nalls M, Simon-Sanchez J. 2017. Lack of evidence for a role of genetic variation in TMEM230 in the risk for Parkinson's disease in the Caucasian population. Neurobiol Aging 50:167 e111-167 e113.

He YC, Huang P, Li QQ, Sun Q, Li DH, Wang T, Shen JY, Chen SD. 2016. TMEM230 stop codon mutation is rare in parkinson's disease and essential tremor in eastern China. Mov Disord.

Hellstrom M, Phng, L.K., Hofmann, J.J., Wallgard, E., Coultas, L., Lindblom, P., Alva, J., Nilsson, A.K., Karlsson, L., Gaiano, N., et al. 2007. D114 signalling through Notch1 regulates formation of tip cells during angiogenesis. Nature 445:776-780.

Hofmann K. 1993. TMbase - A database of membrane spanning proteins segments. Biol Chem Hoppe-Seyler 374(166).

Hogan BM, Herpers, R., Witte, M., Helotera“ , H., Alitalo, K., Duckers, H.J., and Schulte-Merker, S. 2009. Vegfc/Flt4 signalling is suppressed by D114 in developing zebrafish intersegmental arteries. Development 136:4001-4009.

Isogai S, Lawson ND, Torrealday S, Horiguchi M, Weinstein BM. 2003. Angiogenic network formation in the developing vertebrate trunk. Development 130(21):5281-5290.

Kim MJ, Deng HX, Wong YC, Siddique T, Krainc D. 2017. The Parkinson's diseaselinked protein TMEM230 is required for Rab8a-mediated secretory vesicle trafficking and retromer trafficking. Hum Mol Genet.

Kimmel CB, Ballard WW, Kimmel SR, Ullmann B, Schilling TF. 1995. Stages of embryonic development of the zebrafish. Dev Dyn 203(3):253-310.

Lawson ND, Scheer N, Pham VN, Kim CH, Chitnis AB, Campos-Ortega JA, Weinstein BM. 2001. Notch signaling is required for arterial-venous differentiation during embryonic vascular development. Development 128(19):3675-3683.

Lawson ND, Weinstein, B. M. 2002. In vivo imaging of embryonic vascular development using transgenic zebrafish. Dev Biol 248:307-31817.

Leslie JD, Ariza-McNaughton L, Bermange AL, McAdow R, Johnson SL, Lewis J. 2007. Endothelial signalling by the Notch ligand Delta-like 4 restricts angiogenesis. Development 134(5):839-844.

Lobov IB, Renard, R.A., Papadopoulos, N., Gale, N.W., Thurston, G., Yancopoulos, G.D., and Wiegand, S.J. 2007. Delta-like ligand 4 (D114) is induced by VEGF as a negative regulator of angiogenic sprouting. Proc

Natl Acad Sci USA 104:3219-3224. 
Morcos PA. 2007. Achieving targeted and quantifiable alteration of mRNA splicing with Morpholino oligos. Biochem Biophys Res Commun 358(2):521-527.

Nasevicius A, Ekker SC. 2000. Effective targeted gene 'knockdown' in zebrafish. Nat Genet 26(2):216-220.

Olszewska DA, Fearon C, Lynch T. 2016. Novel gene (TMEM230) linked to Parkinson's disease. J Clin Mov Disord 3:17.

Parsons MJ, Pisharath H, Yusuff S, Moore JC, Siekmann AF, Lawson N, Leach SD. 2009. Notch-responsive cells initiate the secondary transition in larval zebrafish pancreas. Mech Dev 126(10):898-912.

Phng LK, Gerhardt H. 2009. Angiogenesis: a team effort coordinated by notch. Dev Cell 16(2):196-208.

Quadri M, Breedveld GJ, Chang HC, Yeh TH, Guedes LC, Toni V, Fabrizio E, De Mari M, Thomas A, Tassorelli C, Rood JP, Saddi V, Chien HF, Kievit AJ, Boon AJ, Stocchi F, Lopiano L, Abbruzzese G, Cortelli P, Meco G, Cossu G, Barbosa ER, Ferreira JJ, International Parkinsonism Genetics N, Lu CS, Bonifati V. 2017. Mutations in TMEM230 are not a common cause of Parkinson's disease. Mov Disord.

Roman BL, Pham VN, Lawson ND, Kulik M, Childs S, Lekven AC, Garrity DM, Moon RT, Fishman MC, Lechleider RJ, Weinstein BM. 2002. Disruption of acvrl1 increases endothelial cell number in zebrafish cranial vessels. Development 129(12):3009-3019.

Schiavone M, Rampazzo E, Casari A, Battilana G, Persano L, Moro E, Liu S, Leach SD, Tiso N, Argenton F. 2014. Zebrafish reporter lines reveal in vivo signaling pathway activities involved in pancreatic cancer. Dis Model Mech 7(7):883-894.

Shutter JR, Scully S, Fan W, Richards WG, Kitajewski J, Deblandre GA, Kintner CR, Stark KL. 2000. Dl14, a novel Notch ligand expressed in arterial endothelium. Genes Dev 14(11):1313-1318.

Siekmann AF, Lawson ND. 2007. Notch signalling limits angiogenic cell behaviour in developing zebrafish arteries. Nature 445(7129):781-784.

Tammela T, Zarkada G, Wallgard E, Murtomaki A, Suchting S, Wirzenius M, Waltari M, Hellstrom M, Schomber T, Peltonen R, Freitas C, Duarte A, Isoniemi H, Laakkonen P, Christofori G, Yla-Herttuala S, Shibuya M, Pytowski B, Eichmann A, Betsholtz C, Alitalo K. 2008. Blocking VEGFR-3 suppresses angiogenic sprouting and vascular network formation. Nature 454(7204):656-660.

Thisse C, Thisse B, Schilling TF, Postlethwait JH. 1993. Structure of the zebrafish snail1 gene and its expression in wild-type, spadetail and no tail mutant embryos. Development 119(4):1203-1215.

Thompson MA, Ransom DG, Pratt SJ, MacLennan H, Kieran MW, Detrich HW, 3rd, Vail B, Huber TL, Paw B, Brownlie AJ, Oates AC, Fritz A, Gates MA, Amores A, Bahary N, Talbot WS, Her H, Beier DR, Postlethwait JH, Zon LI. 1998. The cloche and spadetail genes differentially affect hematopoiesis and vasculogenesis. Dev Biol 197(2):248-269.

Tusnady GE, Simon I. 2001. The HMMTOP transmembrane topology prediction server. Bioinformatics 17(9):849-850. 
Wang HU, Chen, Z. F. and Anderson, D. J. . 1998. Molecular distinction and angiogenic interaction between embryonic arteries and veins revealed by ephrin-B2 and its receptor Eph-B4. . Cell 93:741-753.

Westerfield M. 1993. The zebrafish book. Eugene OUoOP, editor.

Wu H, Zheng X, Cen Z, Xie F, Chen Y, Lu X, Luo W. 2016. Genetic analysis of the TMEM230 gene in Chinese patients with familial Parkinson disease. Parkinsonism Relat Disord.

Wu HH, Brennan C, Ashworth R. 2011. Ryanodine receptors, a family of intracellular calcium ion channels, are expressed throughout early vertebrate development. BMC Res Notes 4:541.

Yan W, Tang B, Zhou X, Lei L, Li K, Sun Q, Xu Q, Yan X, Guo J, Liu Z. 2017. TMEM230 mutation analysis in Parkinson's disease in a Chinese population. Neurobiol Aging 49:219 e211-219 e213.

Yaniv K, Isogai S, Castranova D, Dye L, Hitomi J, Weinstein BM. 2006. Live imaging of lymphatic development in the zebrafish. Nat Med 12(6):711-716.

\section{Figure legends}

Figure 1. Genomic context of the tmem 230 family across selected species and gene structure analysis of zebrafish tmem 230a and tmem230b._(a) The genomic context of zebrafish tmem $230 a$ with its orthologous and paralogous gene tmem $230 \mathrm{~b}$. The gene placed in the center of the panel and aligned over the vertical line is the reference gene used as query (tmem230a). Blue square nodes (left part of the figure) represent ancestral species leading from the same root ancestral species to orthologs and/or paralogs of the gene used as query. Red square nodes represent duplication events of an ancestral version of the gene used as query. Open blue square nodes represent extant species. Upper thicker blue line represents the path leading from the ancestral root to the reference species used as query. Genes indicated by the same color are paralogs (without black boundary) or orthologs (black boundary). The figure was derived from the output of the Genomicus website (version 83.01). (b) Schematic representation of genes tmem230a and tmem230b. 
Exons are indicated by blue boxes (for untranslated sequences) or orange boxes (for coding sequences) and introns are indicated by lines. Intron and exon lengths are not to scale. Lengths are shown in base pairs (bp). Exon-intron structure is derived from the Ensembl Genome Browser.

Figure 2. Spatio-temporal expression of zebrafish tmem 230a. (a) tmem230a is expressed both maternally and zygotically during development. tmem $230 a$ and $\beta$-actin qualitative RT-PCR expression analysis on total RNA from oocytes, and various embryonic and larval stages (from 1-8 cells to $5 \mathrm{dpf}$ ) and adult organs and tissues. Negative control is no cDNA. The sizes of the PCR fragments are indicated. DNA Marker is a $1 \mathrm{~Kb}$ ladder. (b-j) Whole Mount In Situ Hybridization (WISH) of tmem230a during embryo development. (b-d, f and $\mathbf{g}$ ) Lateral views. (b) 15 somite stage embryo (after yolk removal), white arrowhead: forming axial vasculature. (c) 26 hpf embryo and (c') higher magnification of the tail shows the expression in the CV, black arrowhead: CV. (d) Magnification of the head of a $26 \mathrm{hpf}$ embryo, red arrowhead: the pharyngeal arch mesenchyme. (e) Dorsal and (f) lateral magnifications of the head, white asterisks: fin bud, and (g) lateral magnification of the tail of a 2 dpf embryo. Embryos are shown anterior to the left (b-g) Magnifications 40X and 63X. Transverse sections at the level of the trunk (h) and the tail (i) of a 26 hpf embryo, and tail (j) of a 2 dpf embryo. Histological analysis shows the signal in the DA: Dorsal Aorta, PCV: Posterior Cardinal Vein and CV: Caudal Vein. NC: Notochord. Magnification 280X. 
Figure 3. tmem 230a-MO1 injection increases endothelial cell number within the ISVs. (a,b) Bright-field images of 29 hpf embryos injected with (a) std-MO and (b) tmem230a-MO1. Boxed areas indicate the ISVs considered for endothelial cell count. Magnification 40X. (c-d) Confocal fluorescent images of the tail of $\operatorname{tg}(f l i 1: \mathrm{nEGFP})^{\mathrm{y} 7}$ embryos injected with (c) std-MO and (d) tmem230a-MO1. Numbers indicate the cells within an ISV. Cell number is obtained by counting nuclei as the $\operatorname{tg}(f l i 1: \mathrm{nEGFP})^{\mathrm{y} 7}$ embryos display GFP in nuclei of fli1 expressing cells. Magnification 20X. (e) Quantitative analysis of the cell number within the ISVs of $29 \mathrm{hpf}$ $\operatorname{tg}(f l i 1: \mathrm{nEGFP})^{\mathrm{y} 7}$ embryos injected with std-MO and tmem230a-MO1. The number of analysed embryos is indicated by $\mathrm{n} .{ }^{* * *} \mathrm{p}<0.001$ vs std-MO. (f,g) Cross sections of the trunk of $29 \mathrm{hpf}$ embryos injected with (f) std-MO or (g) tmem230a-MO1. NC: NotoChord, DA: Dorsal Aorta, and PCV: Posterior Cardinal Vein. Magnification 350X. Horizontal line with cap is the mean \pm s.e.m.

(h-j) Confocal fluorescent images of the tail of 29 hpf $\operatorname{tg}(f l i l: n E G F P)^{\mathrm{y} 7}$ embryos injected with (h) 1 pmol/embryo of std-MO, (i) 1 pmol/embryo of tmem230a-MO2 or (j) 400 pg/embryo of tmem230a mRNA. Numbers indicate cells within an ISV. (k,l) Quantitative analysis of the cell number within the ISVs in $29 \mathrm{hpf} \operatorname{tg}(f l i 1: \mathrm{nEGFP})^{\mathrm{y} 7}$ embryos injected with (k) std-MO, tmem230a-MO1 or tmem230a mRNA, or with (l) std-MO, or tmem 230a-MO2 on its own or in combination with tmem230a mRNA. The number of injected embryos analysed is $\mathrm{n} .{ }^{* * *} \mathrm{p}<0.001$ vs std-MO (k) and $* * * \mathrm{p}<0.001$ vs MO2 (l). Horizontal line with cap is the mean \pm s.e.m. Magnification $20 \mathrm{X}$. 


\section{Figure 5. tmem 230a acts synergistically with the Dll4/Notch pathway in regulating} ISV cell number. (a-d) Confocal fluorescent images of the tail of $29 \mathrm{hpf}$ $\operatorname{tg}(\text { fli1:nEGFP })^{\mathrm{y} 7}$ embryos injected with (a) $0.4 \mathrm{pmol} / \mathrm{embryo}$ of std-MO; (b) 0.4 pmol/embryo of dll4-MO; (c) $0.07 \mathrm{pmol} / \mathrm{embryo}$ (subcritical dose) of tmem230a-MO1 together with $0.09 \mathrm{pmol} / \mathrm{embryo}$ (subcritical dose) dll4-MO; and (d) $400 \mathrm{pg} / \mathrm{embryo}$ of tmem $230 a$ mRNA together with $0.4 \mathrm{pmol} /$ embryo dll4-MO. In a-d numbers indicate the cells within a representative ISV. Magnification 20X. (e-f) Quantitative analysis of the cell number within the ISVs of $29 \mathrm{hpf} \operatorname{tg}(f l i 1: \mathrm{nEGFP})^{\mathrm{y} 7}$ embryos. (e) Embryos were injected with std-MO, or subcritical dose of tmem230a-MO1, or subcritical dose of dll4MO, or subcritical dose of tmem230a-MO1 together with subcritical dose of dll4-MO. *** $\mathrm{p}<0.001$ vs std-MO, vs tmem230a-MO1 and vs dll4-MO. (f) Embryos were injected with $0.4 \mathrm{pmol} / \mathrm{embryo}$ dll4-MO on its own and together with tmem $230 a$ mRNA. *** $\mathrm{p}<0.001$ vs dll4-MO. (g) Embryos were injected with std-MO or tmem230a mRNA and then treated with DAPT. ${ }^{* * *} \mathrm{p}<0.001$ vs std-MO + DAPT. Control embryos (std-MO) and tmem230a mRNA injected embryos were treated with the same concentration of DMSO as DAPT immersed embryos. The number of injected embryos analysed is $n$. Horizontal line with cap is the mean \pm s.e.m. 
Figure 6. tmem 230a-MO1 injection inhibits Notch signalling activation in vessels. (ad) Analysis of mCherry expression in vessels of $48 \mathrm{hpf}$ embryos derived from the transgenic line $\operatorname{tg}(\mathrm{T} 2 \mathrm{KTp} \text { lbglob:hmgb1-mCherry })^{\mathrm{jh} 11}$ crossed with the transgenic line $\operatorname{tg}(f l i 1 \text { :EGFP })^{\mathrm{y} 1}$; injected with std-MO (a,b) or tmem230a-MO1 (c,d). ISV: InterSomitic Vessel, and DA: Dorsal Aorta. Magnification 40X.

\section{Supplementary Figure 1. Tmem230 protein analysis.}

(a) Amino acid identity and similarity (in parentheses) between zebrafish (Danio rerio) Tmem230a and Tmem230b and human and mouse ortholog proteins: Homo sapiens (gi:42476068) and Mus musculus (gi:213972600). (b) Topology prediction analysis of conserved domains revealed that Tmem230a and Tmem230b proteins contain 2 transmembrane domains.

\section{Supplementary Figure 2. Spatio-temporal expression pattern of zebrafish tmem $230 b$.}

(a) tmem $230 b$ and $\beta$-actin qualitative RT-PCR expression analysis on total RNA from oocytes, and embryonic and larval stages (from 1-8 cells to $5 \mathrm{dpf}$ ) and different adult 
organs and tissues. DNA Markers are 100 bp ladder and $1 \mathrm{~Kb}$ ladder. Negative control is no cDNA. The sizes of the PCR fragments are indicated. tmem $230 b$ is expressed both maternally and zygotically in embryo development. (b-f) WISH analysis of tmem $230 b$ at various developmental stages. (b) Lateral view of a 15 and of a (c) 20 somite stage embryo, white arrowhead: somites. (d) Lateral view of a 26 hpf embryo and (d') higher magnification of the tail shows the expression of tmem $230 \mathrm{~b}$ in the $\mathrm{CV}$ region and somites, red arrowhead: somites and black arrowhead: CV region. Head at (e) $26 \mathrm{hpf}$ and at (f) 2 dpf. Embryos are shown anterior to the left. Magnifications 40X and 63X.

\section{Supplementary Figure 3. MO1 and MO2 are designed to specifically target the tmem 230a transcript.}

(a) Sequence alignments obtained with CLUSTAL W, between tmem230a and tmem230b, tmem $230 a$ and tmem $230 b$ with the sequences of tmem230a-MO1 and tmem230a and tmem230b with tmem230a-MO2 demonstrate the specificity of the MOs with the tmem230a sequence. Green box shows translation start sites. (b) Schematic representation of the position of tmem230a-MO1 and tmem230a-MO2 binding to tmem $230 a$ mRNA. Exon and intron lengths are indicated. The effectiveness of the spliceblocking morpholino, tmem 230a-MO2 designed to target exon 2 was shown by the generation of an amplification product that excludes exon 2 using forward (For.) and reverse (Rev.) primers designed in the first and last exons. Injection of splice-blocking tmem230a-MO2 results in the generation of a smaller product (in red box) corresponding to the tmem $230 a$ transcript lacking exon 2 . The sizes of the PCR fragments are indicated. DNA Marker is a $1 \mathrm{~Kb}$ ladder. 


\section{Supplementary Figure 4. Dose-response of tmem 230a-MO1 on embryo survival and morphological defects and dose-response of tmem 230a-MO2 on ISV cell number.}

(a) Survival and morphological defects (bent tail and small head) histograms of $\operatorname{tg}(f l i 1: n E G F P)^{\mathrm{y} 7}$ embryos at 29 hpf injected with different doses of tmem230a-MO1. (b) Quantitative analysis of cell number in the ISVs for $\operatorname{tg}(f l i 1: n E G F P)^{\mathrm{y} 7}$ embryos injected with std-MO and different doses of tmem230a-MO2 at 29 hpf. The number of injected embryos is $\mathrm{n} . * * \mathrm{p}<0.01$ vs std-MO.

\section{Supplementary Figure 5.}

tmem 230a-MO1 injection causes no gross effects on vasculogenesis and angiogenesis at 2 dpf. In vivo analysis of $\operatorname{tg}\left(f l i 1\right.$ :EGFP) ${ }^{\mathrm{y} 1}$ embryos injected with (a-d) std-MO or (e-h) tmem230a-MO1. (a,b,e and f) Bright field and (c,d,g and h) fluorescence images. Magnification of the caudal region (b,d,f, and $\mathbf{h})$. Lateral views are shown anterior to the left. DLAVs: Dorsal Longitudinal Anastomotic Vessels, ISVs: Intersomitic Vessels, DA: Dorsal Aorta, and CV: Caudal Vein. Magnifications 25X and 63X. 
a

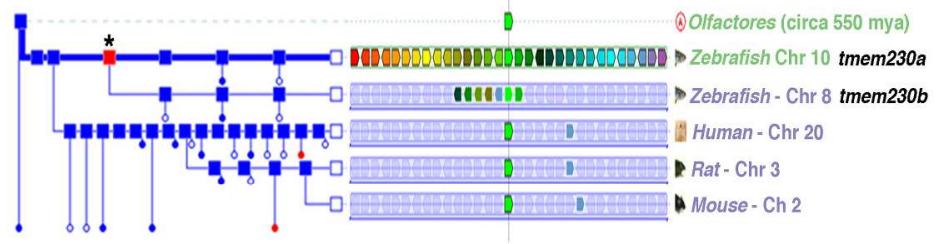

b

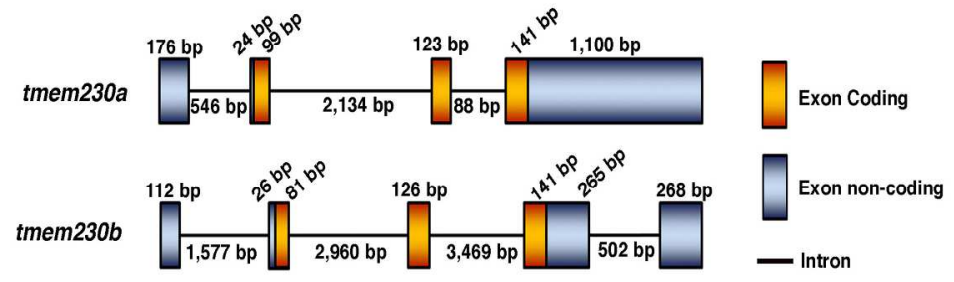

Figure 1. Genomic context of the tmem 230 family across selected species and gene structure analysis of zebrafish tmem230a and tmem230b.

Figure 1 . Genomic context of $\mathrm{t}$

$254 \times 317 \mathrm{~mm}(300 \times 300 \mathrm{DPI})$ 
Figure 2

a
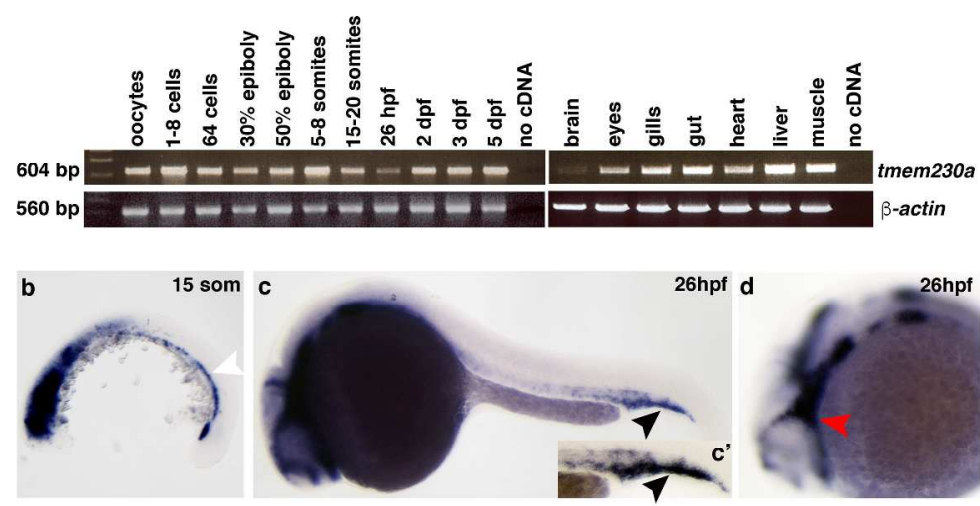

e 2 dpf $f$

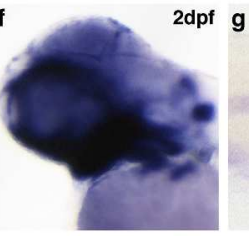

2dpf

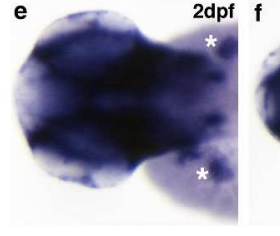

p $g$
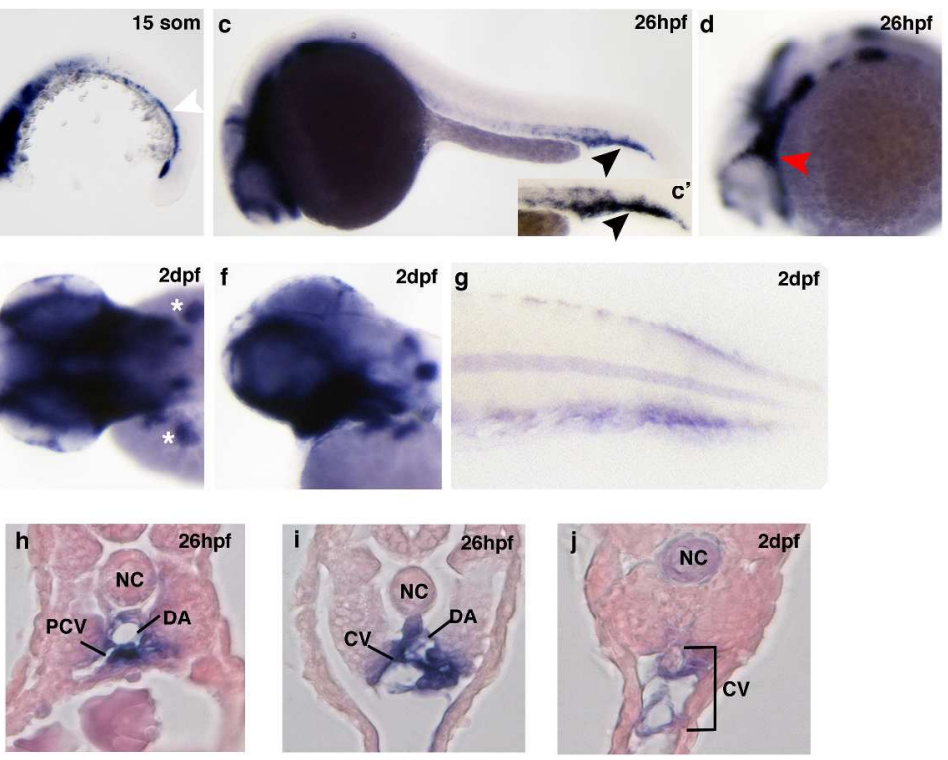

Figure 1. Genomic context of the tmem230 family across selected species and gene structure analysis of zebrafish tmem230a and tmem230b.

Figure 1. Genomic context of $t$

$275 \times 361 \mathrm{~mm}(300 \times 300$ DPI $)$

John Wiley \& Sons, Inc. 
Figure 3
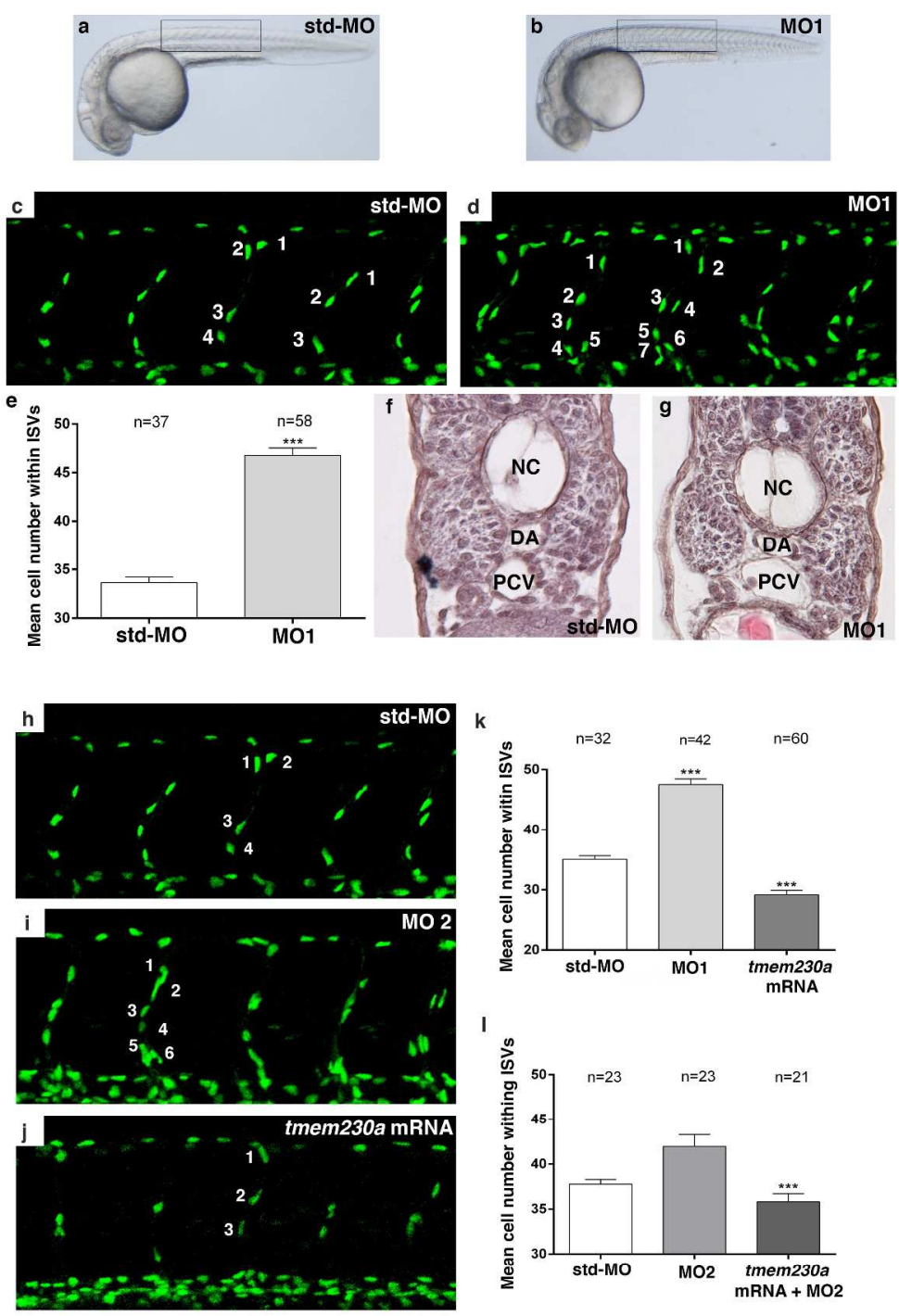

Figure 3. tmem230a-MO1 injection increases endothelial cell number within the ISVs. Figure 3. tmem230a-MO1 injecti $297 \times 420 \mathrm{~mm}(300 \times 300$ DPI) 


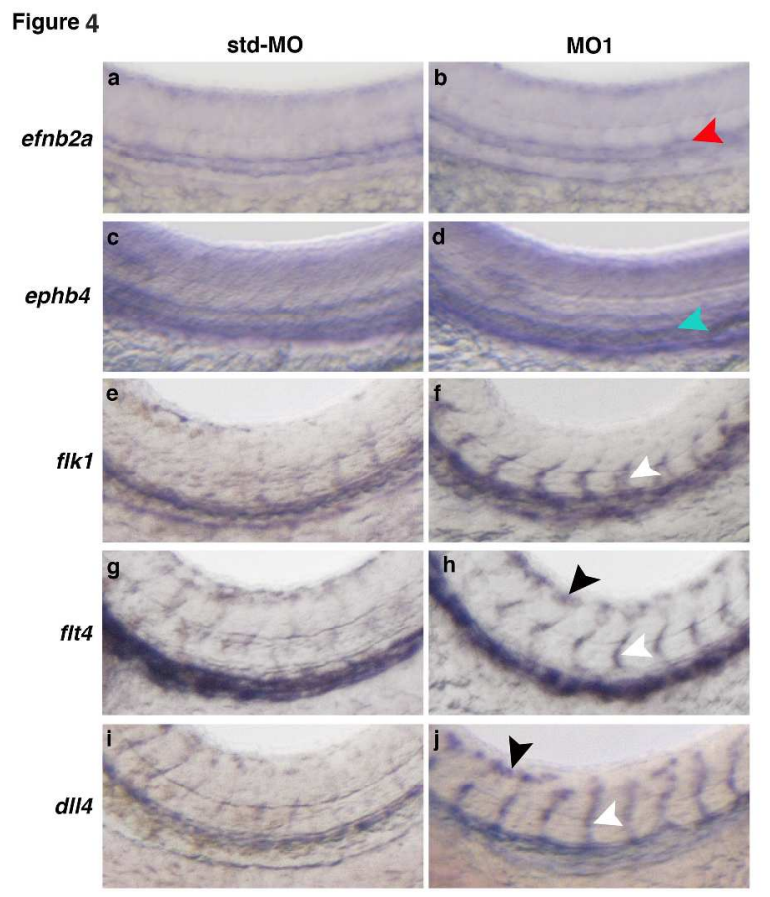

Figure 4. tmem230a morphants display an increase of tip cell markers.

Figure 4. tmem230a morphants $d$ $297 \times 420 \mathrm{~mm}(300 \times 300 \mathrm{DPI})$

John Wiley \& Sons, Inc. 
Figure 5. tmem230a acts synergistically with the DII4/Notch pathway in regulating ISV cell number. Figure 5. tmem230a acts synerg

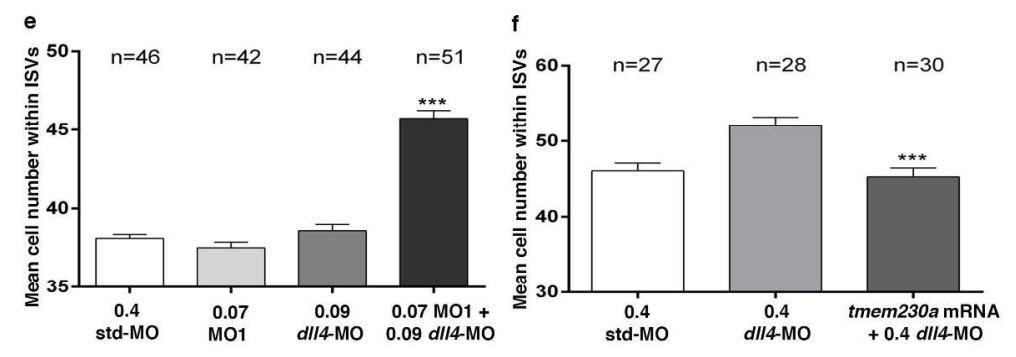

Figure 5
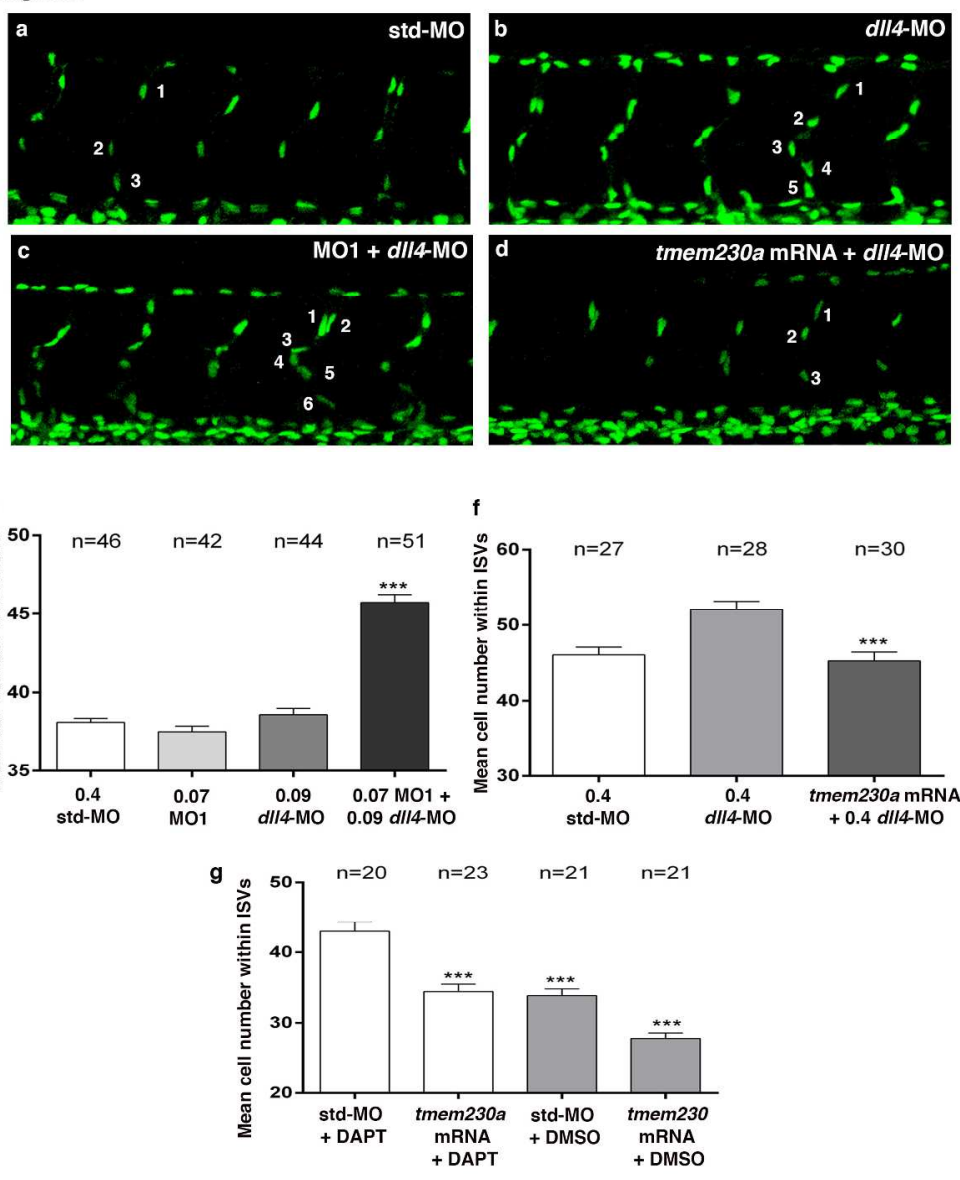

$209 \times 297 \mathrm{~mm}(300 \times 300 \mathrm{DPI})$ 

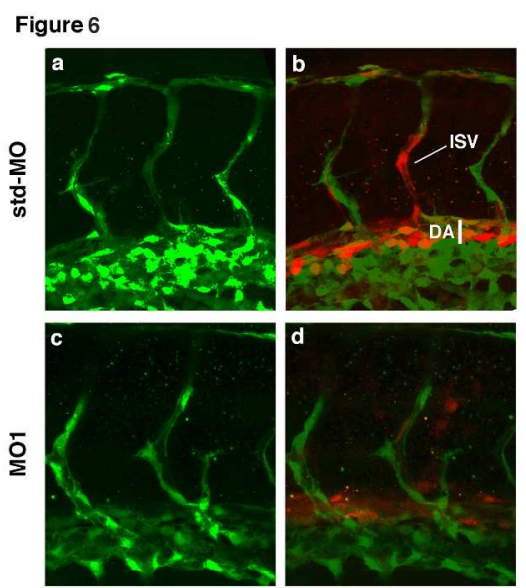

Figure 6. tmem230a-MO1 injection inhibits Notch signalling activation in vessels.

Figure 6. tmem230a-MO1 injecti $209 \times 297 \mathrm{~mm}(300 \times 300 \mathrm{DPI})$

John Wiley \& Sons, Inc. 